\title{
Product Certification by Automated Microscopy in an Industrial Setting
}

\author{
T.C. Eck, ${ }^{*}$ W. Soufi, ${ }^{* *}$ A. Nasrallah**
}

*Alcoa Mill Products, PO Box 3167, Lancaster PA, 17604

**Novovision, Inc., 301 N Harrison St. Suite 384, Princeton NJ, 08540

System Overview:

Clad aluminum sheet composite samples are measured and evaluated and the corresponding lots certified or rejected, all without operator intervention.

A technician prepares a large metallographic pack-mount containing tens to hundreds of samples, places it on the metallograph stage, and enters the contents in a data entry interface.

The system compares each item to its specification using the modularly defined image type for that item to define the layers.

When all required sample sites for a lot have been inspected and passed, the system sends the data to the mainframe computer for lot certification.

Other actions taken by the system include printing rejections, printing a delayed materials report, or printing unexpected condition reports.

The Product:

Alcoa, Lancaster produces about a thousand different clad sheet products. These are aluminum sheet with different alloys metalurgically bonded in layers. Most are for automotive heat exchangers and include at least one low-melting layer for brazing. Other layers may be present to inhibit corrosion, increase strength or facilitate brazing.

Imaging Methods:

The redundancy due to the parallel nature of the layers being measured allows for exceptional system robustness while still allowing data verification. The camera and motorized stage axes are aligned since both are used for measurement purposes. The image is trisected perpendicular to the layer interfaces and each sub-image is measured independently (the measurement is discarded if the three sub-images yield different results). Each sub-image is collapsed along one axis yielding a linear array. This is typically accomplished by averaging the values but other functions such as standard deviation may be used to create the linear arrays. FIG. 2.

At this point, the system looks up the product number in a database for the image type, gauge, and inspection criteria. The image type defines the interfaces in terms of maxima or minima of certain numerical characteristics, starting with the most distinct and working toward the faintest. In this way, very faint interfaces can be found by looking only between better-defined ones. FIG. 3. Total image processing time is less than $100 \mathrm{msec}$. The numerical characteristics of most use are:

1 Standard deviation or variance (max)

2 Differential operator (max and $\min$ )

3 Brightness (min)

All except brightness are defined over an interval in the linear array that is set depending on the distinctness of the interface in that image type - a function of magnification and various metallurgical characteristics. The system measures each sample in this manner, moving the stage the nominal gauge to find the next sample (plus an algebraic increment determined from the amount the prior image was off center). System robustness allows all decision points to be automated. 


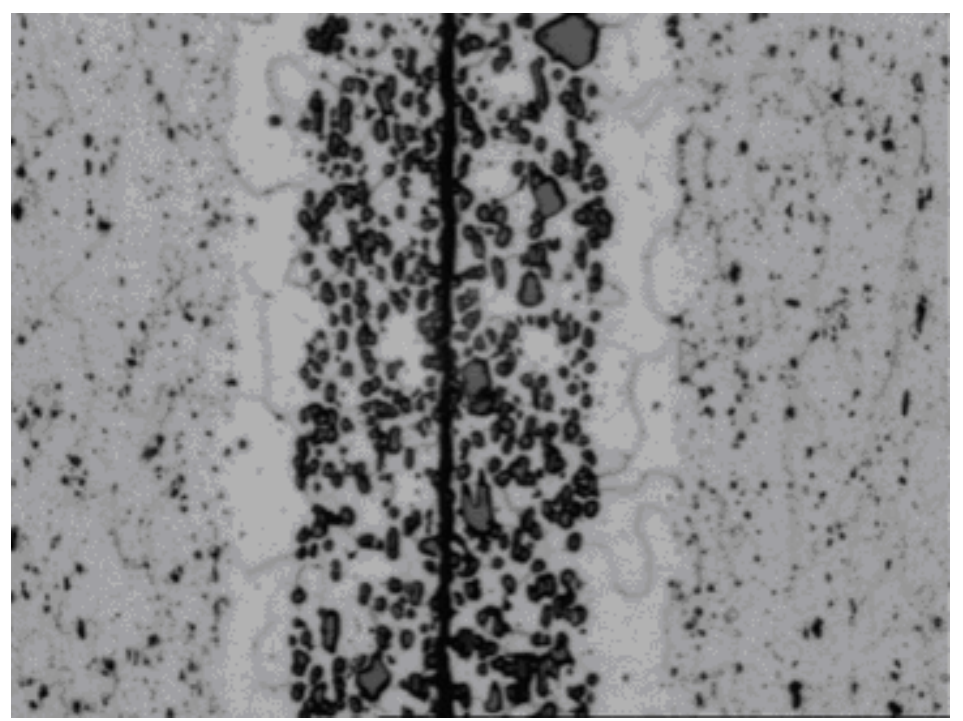

FIG. 1. Five layer clad products centered at sample interface.

PRofle

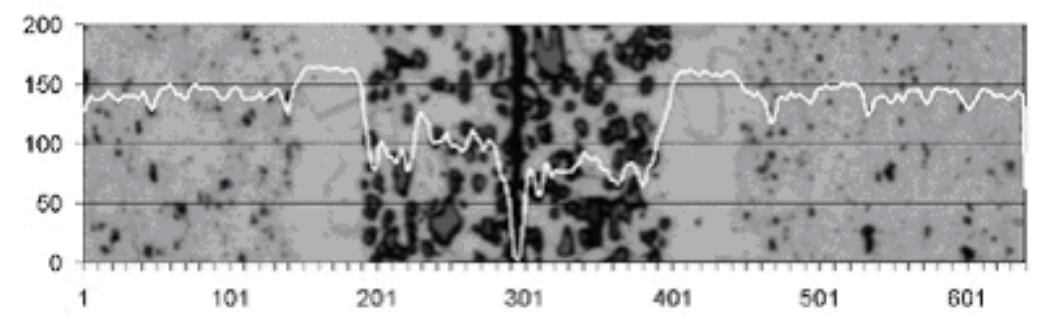

FIG. 2. Bottom third of image with brightness profile.

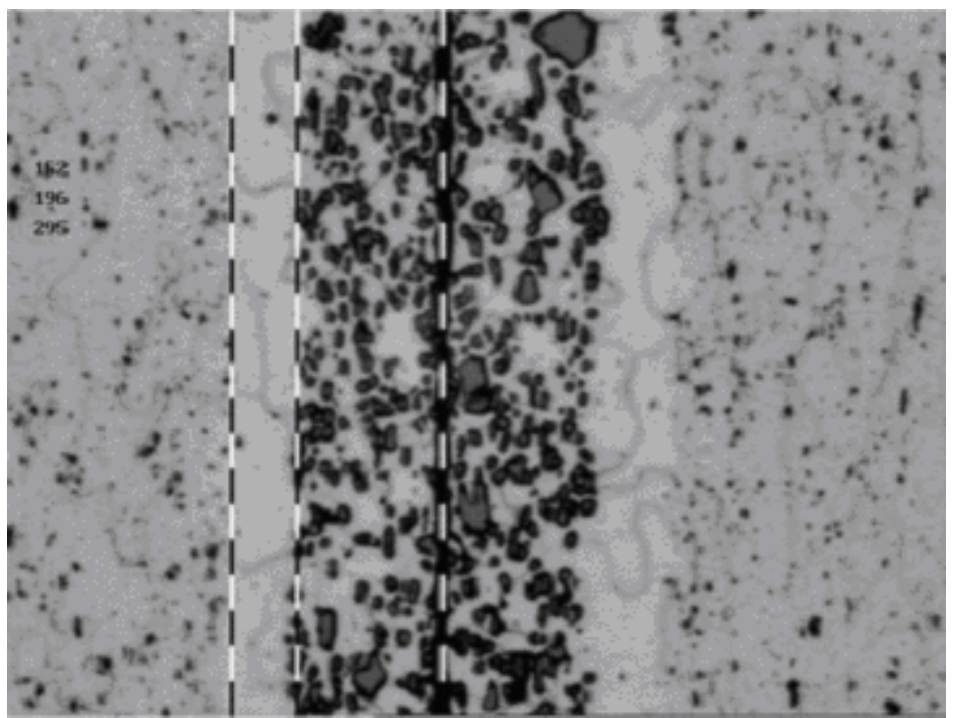

FIG. 3. Liner interfaces found in right to left order as 1) brightness minimum of entire image, 2) Differential minimum from left side to interface 1,3) Differential maximum from left side to interface 2. 